\title{
Quantitative tests of mixed crystal exciton theory. II. Energy denominator study of naphthalene ${ }^{1} B_{2 u}$ resonance pairs*
}

\author{
Frederick W. Ochs ${ }^{\dagger}$ and Raoul Kopelman \\ Department of Chemistry, The University of Michigan, Ann Arbor, Michigan 48109 \\ (Received 29 October 1975)
}

A spectroscopic absorption study is presented of 1- $D_{1} C_{10} H_{7}, 2-D_{1} C_{10} H_{7}$, and 1,4- $D_{2} C_{10} H_{7}$ resonance pairs in $\mathrm{C}_{10} \mathrm{D}_{8}$ over a concentration range of $0.009 \%$ to $5 \%$ mole, at $2{ }^{\circ} \mathrm{K}$, with a resolution of $0.5 \mathrm{~cm}^{-1}$. The resulting structure is interpreted in terms of the pairwise exciton exchange interactions, $M_{1 / 2(a+b)}, M_{a}, M_{b}$, $\boldsymbol{M}_{c}, \boldsymbol{M}_{a+c}$, and $\boldsymbol{M}_{1 / 2(a+b)+c}$. The energy denominator dependence is interpreted in terms of both exciton quasiresonance and exciton superexchange. The three parameter sets derived by Hong and Kopelman to fit earlier data on neat and isotopic mixed crystals are corroborated by this study, as they were in our previous study on monomers (I). However, it was not possible to narrow down completely the choice of parameters. A discussion is given on the feasibility of deriving a unique dispersion relation (and Green's function) for the first naphthalene singlet exciton and on its likely precision.

\section{INTRODUCTION}

In a previous article (henceforth called I), we have successfully related quantitative experiments involving isolated isotopic naphthalene guest molecules ("monomers") in a host lattice, with existing theory. In that work excitation-exchange interactions were utilized in calculating the pure crystal Green's function. From this pure crystal Green's function we derived excellent theoretical predictions of experimental measurables like quasiresonance shifts (determining the energies of the monomer guests), spectral polarization ("Rashba effect"), and the degree of the exciton localization at the impurity (monomer) site. However, we did not fully elaborate there on the excitation-exchange parameters, which are obviously of paramount importance in molecular crystals; that is, we did not discuss the recent, most crucial experimental tests to which these parameters have been exposed so far. These tests consist of an energy denominator investigation of naphthalene resonance pairs ("dimers") and are described in the present paper.

We assume that in the deep trap limit the resonance pair interaction represents (within experimental error) the pairwise exciton exchange interaction between two molecules of the same relative orientation and displacement in the pure crystal. ${ }^{2-7}$ In the lowest excited electronic states of the naphthalene crystal, as well as in other exciton states of naphthalene and other molecular crystals where the longer-range transition-dipoletransition-dipole interactions are negligible, the exciton exchange interactions fall off rapidly with distance so that only a few near-neighbor interactions suffice to characterize the pure crystal exciton states to an accuracy sufficient to both justify the restricted DavydovFrenkel limit ${ }^{7-10}$ and to predict properties such as pure crystal band structure, quasiresonance shifts, delocalization, etc. ${ }^{1}$ Because of this short-range nature of interactions, only a few "resonance pairs" should be spectroscopically observable; the more distant interactions being too low in energy to form pair levels resolvable from the isolated guest ("monomer") level. This is precisely what has been observed by Hanson, ${ }^{3}$
Broude, and Leiderman, ${ }^{11}$ Hong and Robinson, ${ }^{12}$ and by Braun and Wolf ${ }^{13}$ in both the first excited singlet and triplet states. However, one must consider the fact that naphthalene $\mathrm{H}_{8}$ in $\mathrm{D}_{8}$ is not a deep trap (trap depth $\Delta \approx-115 \mathrm{~cm}^{-1}$, bandwidth $B \approx 160 \mathrm{~cm}^{-1}$ ) and therefore interactions with the host cannot be neglected. ${ }^{4,5}$ From the resonance pair splittings (relative to the isolated guest) obtained by Hanson, ${ }^{3}$ Hong and Kopelman ${ }^{5}$ were able to correct for the guest-host (actually guest-hostsguest) interactions, and to abstract sets of corrected values of the resonance pair splittings (Table I). The amount by which the splitting is corrected has been called the exciton superexchange interaction. This is an effect which is in addition to the quasiresonance effect.1,14,15 Originally six sets were derived, due to the fact that six arbitrary assignments can be made for the pair spectra which are observed. ${ }^{5}$ However, only the three sets listed in Table I give satisfactory agreement with the experimentally measured exciton band. ${ }^{16}$ Hong and Kopelman ${ }^{5}$ pointed out that the relative quality of these sets of parameters could be further tested by a study on the trap-depth dependence of the uncorrected (i.e., observed) pair splitting.

The results of our study are both disappointing and encouraging. No significant further refinement of the

TABLE I. Excitation exchange interaction parameters for the ${ }^{1} B_{2 u}$ state of naphthalene.

\begin{tabular}{lrrr}
\hline \hline & & \multicolumn{3}{c}{$M^{\mathrm{b}}\left(\mathrm{cm}^{-1}\right)$} \\
Position $^{2}$ & Set 1 & Set 2 & Set 3 \\
\hline $1 / 2(a+b)^{\mathrm{c}}$ & 18.0 & 18.0 & 18.0 \\
$1 / 2(a+b)+c^{\mathrm{c}}$ & 2.0 & 1.0 & 1.0 \\
$a^{\mathrm{d}}$ & -0.6 & -4.3 & -1.2 \\
$b^{\mathrm{d}}$ & -3.9 & 1.9 & 1.6 \\
$c^{\mathrm{d}}$ & 6.1 & -6.1 & -8.9 \\
$a+c^{\mathrm{d}}$ & -3.7 & 6.0 & 6.0 \\
\hline \hline
\end{tabular}

${ }^{2}$ Position of the second molecule of the pair with respect to the first.

${ }^{0}$ Pairwise interaction, Hong and Kopelman. ${ }^{5}$

${ }^{c}$ Interchange equivalent pairs.

${ }^{\mathrm{d}}$ Translationally equivalent pairs. 
pairwise exciton interaction parameters was possible. However, we find that our previously accepted exciton density-of-states and exciton dispersion relationships, as well as the underlying concepts, successfully met a very stringent experimental consistency test.

\section{THEORETICAL BACKGROUND}

Once again, the time independent Green's function formalism has been used to approach this problem. An exact form of the Dyson equation is given by Hong and Kopelman, ${ }^{5}$

$$
\langle G(\mathbf{k})\rangle=G_{0}(\mathbf{k})+G_{0}(\mathbf{k}) \Sigma(\mathbf{k})\langle G(\mathbf{k})\rangle,
$$

where $\langle G(\mathbf{k})\rangle$ is the a veraged mixed crystal Green's function, $G_{0}(\mathbf{k})$ is the pure crystal Green's function, and $\Sigma(\mathbf{k})$ is the self-energy. [Please note that in the dilute crystal situation, discussed in $\mathrm{I}$, as the concentration $\rightarrow 0 \Rightarrow \Sigma(\mathbf{k}) \rightarrow \Delta$ ]. Considering now pairs which are isolated from other guest molecules, one can find the selfenergy operator by summing interactions between the two molecules making up the pair by a technique developed by Yonezawa and Matsubara. ${ }^{17}$ Instead of going into the details of the calculation of the self-energy, we present only the important results (for a more complete derivation see Hong and Kopelman, ${ }^{5}$ and Yonezawa and Matsubara ${ }^{17}$ ). For translationally equivalent pairs, ${ }^{5}$

$$
\Sigma_{2}(\mathbf{k}=0)=\frac{\Delta C^{2}}{\left[1-(\Delta / N) f_{1}(\mathbf{0})\right]-(\Delta / N) f_{1}\left(\mathbf{R}_{e}\right)},
$$

where

$$
f_{\mathrm{I}}(\mathbf{R})=\sum_{\mathbf{k}^{+}} \exp \left(i \mathbf{k}^{+} \cdot \mathbf{R}\right) G_{0}\left(\mathbf{k}^{+}\right)+\sum_{\mathbf{r}} \exp \left(i \mathbf{k}^{-} \cdot \mathbf{R}\right) G_{0}\left(\mathbf{k}^{-}\right) ;
$$

$\mathrm{k}^{+}$belongs to the $A$ branch and $\mathrm{k}^{-}$belongs to the $B$ branch of the interchange group ${ }^{18} C_{2}$,

$$
G_{0}\left(\mathbf{k}^{ \pm}\right)=\left[E-E\left(\mathbf{k}^{ \pm}\right)\right]^{-1},
$$

$\Delta$ is the trap depth, $C$ is the concentration, and $N$ is the number of molecules in the crystal. $\mathbf{R}_{e}$ is the vector of the translational displacement of the second molecule in the pair relative to the first. This form [Eq. (2)] is the value near the pole corresponding to the optically allowed $(+)$ dimer transition. Only one such $(\mathbf{k}=0)$ transition is allowed, since the dipole moments of the out-of-phase combination cancel. ${ }^{3}$ Please note that ${ }^{5} f_{1}(0) / N$ is just the neat crystal Green's function while $f_{1}\left(\mathbf{R}_{e}\right) / N$ is the off-diagonal element of the Green's function between the two molecules. The energy of the observed dimer state is then found by solving

$$
0=\left[1-(\Delta / N) f_{1}(0)\right]-(\Delta / N) f_{1}\left(\mathbf{R}_{e}\right),
$$

namely:

$$
f_{1}(\mathbf{0}) / N+f_{1}\left(\mathbf{R}_{e}\right) / N=1 / \Delta .
$$

The Rashba effect can be calculated ${ }^{5}$ for such a pair transition and it is found to be identical to that of an isolated guest at the dimer energy [Eq. (3) of I].

For pairs which are related by an interchange operation, two transitions should be observed, and the selfenergies near the poles in these two cases are

$$
\Sigma_{2}\left(\mathbf{k}^{+}=0\right)=\frac{\Delta C^{2}}{\left[1-(\Delta / N) f_{1}(0)\right]-(\Delta / N) f_{2}\left(\mathbf{R}_{i}\right)}
$$

and

$$
\Sigma_{2}\left(\mathbf{k}^{-}=0\right)+\frac{\Delta C^{2}}{\left[1-(\Delta / N) f_{1}(0)\right]+(\Delta / N) f_{2}\left(\mathbf{R}_{i}\right)},
$$

$\mathbf{R}_{\boldsymbol{i}}$ is the displacement of the second (interchange equivalent) molecule relative to the first and

$$
f_{2}\left(\mathbf{R}_{\mathbf{i}}\right)=\sum_{\mathbf{k}^{+}} \exp \left(i \mathbf{k}^{+} \cdot \mathbf{R}_{\boldsymbol{i}}\right) G_{0}\left(\mathbf{k}^{+}\right)-\sum_{\mathbf{k}^{-}} \exp \left(i \mathbf{k}^{-} \cdot \mathbf{R}_{\boldsymbol{j}}\right) G_{0}\left(\mathbf{k}^{-}\right) .
$$

Therefore the transition energies are determined by the equations

$$
\text { at } E^{+}:\left[1-(\Delta / N) f_{1}(0)\right]-(\Delta / N) f_{2}\left(\mathbf{R}_{i}\right)=0
$$

and

$$
\text { at } E^{-}: 1-(\Delta / N) f_{1}(\mathbf{0})+(\Delta / N) f_{2}\left(\mathbf{R}_{i}\right)=0 .
$$

The solutions of the above are

$$
f_{1}(\mathbf{0}) / N \pm f_{2}\left(\mathbf{R}_{i}\right) / N=1 / \Delta .
$$

From this we can see that there should be two transitions displaced from the monomer and completely polarized ( $\| \mathrm{b}$ and $\perp b$ ). Again an ana log "Rashba effect" (polarization ratio for doublet) can be calculated and found to be $\mathrm{b}^{5}$

$$
P_{\text {guest }}=P_{\text {host }} \frac{\left[E_{-}-E_{a c}\right]^{2}}{\left[E_{+}-E_{b}\right]^{2}} \frac{d E_{+} / d \Delta}{d E_{-} / d \Delta} .
$$

This also reduces to the form of the isolated guest if $E_{-}-E_{+} \rightarrow 0$.

From the above expressions [(5a) and (8)] for the dimer state energies, it is clear that the resonance pair splittings are not, in general, equal to the exchange interaction energies. The above formalism correctly predicts that the equality holds only in the deep trap limit.

\section{EXPERIMENTAL}

The materials, their purification, and the analytical control tests are described in Ref. 1 , as well as the growing of single crystals and their orientation. The same holds for the cryogenics used to achieve $1.6^{\circ} \mathrm{K}$, as well as the details of the spectroscopic procedures and the computer processing of the photoelectric data. We just add here some details on the photographic procedure, which was used occasionally. Kodak SA-1 film was used. The traces (Fig. 1) were run on a JoyceLoebl Mk. IIIB microdensitometer. Optimum resolution was $0.5 \mathrm{~cm}^{-1}$. Crystal thicknesses were about 0.5 to $1 \mathrm{~mm}$

\section{SPECTROSCOPIC RESULTS}

In Fig. 1 are the spectra of $\alpha D_{1}$ dissolved in $D_{8}$ at various increasing concentrations. At the lowest concentration ( $0.09 \%$ mole) only six absorptions in the region of $\alpha D_{1}$ are seen. According to the results presented in the previous work (I), Hanson's work, ${ }^{3}$ and the mass spectral results, these peaks are assigned to the monomer levels of $\mathrm{C}_{10} \mathrm{H}_{8},{ }^{13} \mathrm{C}^{12} \mathrm{C}_{9} \mathrm{H}_{8}, 1-\mathrm{DC}_{10} \mathrm{H}_{7}$, 1-D ${ }^{13} \mathrm{C}^{12} \mathrm{C}_{9} \mathrm{H}_{7}, 1,4-\mathrm{D}_{2} \mathrm{C}_{10} \mathrm{H}_{6}$ and $1,4-\mathrm{D}_{2}{ }^{13} \mathrm{C}^{12} \mathrm{C}_{9} \mathrm{H}_{6}$, in order of increasing energy. As the concentration in- 


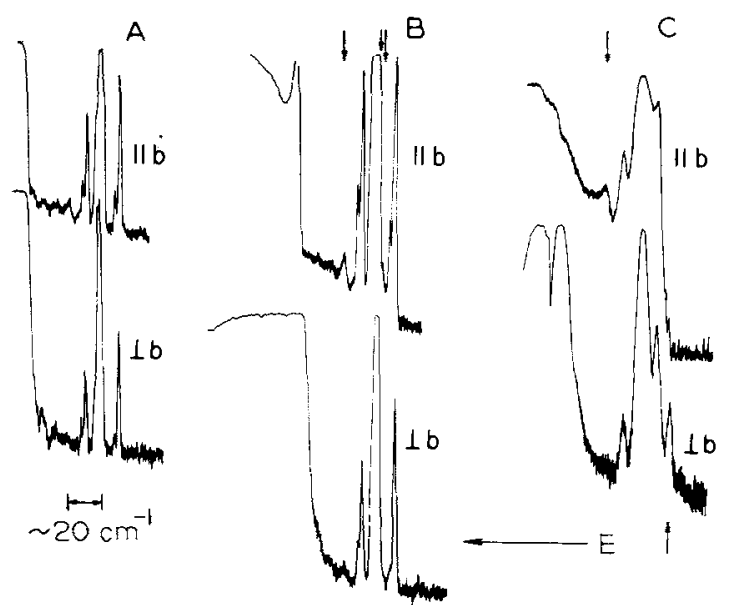

FIG. 1. Polarized absorption spectra of $1-D_{1} C_{10} H_{7}$ in $C_{10} D_{8}$ as a function of concentration. The spectra were recorded photographically at a resolution of $0.7 \mathrm{~cm}^{-1}$. The concentrations of $\alpha \mathrm{D}_{1}$ in the crystals were $0.088 \%, 0.7 \%$, and $5 \%$ mole in $a-c$, respectively. The sharp emission feature in the host band in spectrum $c$ ( $\perp$ b pol.) is a calibration line. The energy in each of these spectra increases in going right to left. The vertical arrows designate pair states (one or two are hard to see on the reproduction).

creases, the intensity of any absorptions due to resonance pairs should increase as the concentration squared, ${ }^{3}$ and this can be compared to the more linearly dependent intensity of the monomer absorption. At $0.7 \%$ concentration of $\alpha \mathrm{D}_{1}$ one can see three additional features appearing at energies of $-2.2,-4.1$, and +18 $\mathrm{cm}^{-1}$ from the $\alpha \mathrm{D}_{1}$ origin. (The first is considerably easier to observe on the film.) The peak at $18 \mathrm{~cm}^{-1}$ is polarized only along the $b$ crystal axis (see Fig. $2 \mathrm{c}$ for a better example of this polarization) while the other two absorptions are stronger in the $b$ polarization than in the $\perp b$. In the yet more concentrated $5 \%$ crystal, one more absorption is observed at $-14.1 \mathrm{~cm}^{-1}$, only in this case it is polarized $\perp$ b. Similar observations were made by Hanson ${ }^{3}$ on $\mathrm{H}_{8}$ in $\mathrm{D}_{8}$. We are relying heavily on his results as an aid in interpreting the se spectra. The important distinction is in the strongly $b$ polarized absorption which Hanson ${ }^{3}$ did not observe in $H_{8}$ in $D_{8}$ because of interference due to absorption by a small concentration of $2-\mathrm{DC}_{10} \mathrm{H}_{7}$ which occurs at +15 $\mathrm{cm}^{-1}$ relative to $H_{8}$. This transition is different from the other monomer and pair transitions in all of these systems in its broad and diffuse character. The exact energy of this peak is therefore quite uncertain compared to the other absorption peaks. We feel then that we have identified four pair transitions in this system as contrasted to the five which Hanson ${ }^{3}$ found in the $\mathrm{H}_{8}$ case. These results are displayed in Table II. The polarizations of the various transitions indicate the type of pair state involved, as mentioned in the section on theoretical background. Therefore the sharply polarized absorptions (at 18 and $-14 \mathrm{~cm}^{-1}$ ) are due to the $1 / 2(a+b)$ interchange pair, and the less sharply polarized lines are due to translational pairs. However, it is difficult to assign the particular translational pair to a particular transition $\left(-2.2\right.$ and $\left.-4.1 \mathrm{~cm}^{-1}\right)$. This same basic analysis was applied to the $\beta D_{1}$ and $\alpha D_{2}$ systems. Actually these latter two are quite similar in appearance, as might be expected, since their trap depths differ by only $2 \mathrm{~cm}^{-1}$. The results for $\beta D_{1}$ are shown in Fig. 2 and those for $\alpha \mathrm{D}_{2}$ are in Fig. 3. The $\perp$ b polarized interchange pair in the $\beta D$ crystals lies almost at the same energy as the $\mathrm{H}_{8}$ absorption. The presence of this transition is indicated by the broadening of the $\mathrm{H}_{8}$ line in the $\perp$ b spectrum (Figs. 2c and 2d) as well as by the greater than expected intensity in the $\perp \mathbf{b}$ absorption compared to the $\| \mathbf{b}$ absorption (see Figs. ?a and $2 \mathrm{~b})$.

We would like to comment on the quality of the calibration of these spectra. Even though the standard deviation in the dispersion fit is on the order of $0.5 \mathrm{~cm}^{-1}$ in this region, it is important to realize that calibration is less accurate when those lines to be calibrated are not well resolved and therefore effectively shifted. Thus the calibration of the pair transitions which are very close to the monomer is undoubtedly less accurate than might be desired. Nor will increased resolution help to a great extent, because of the intrinsic width of monomer transition at the concentrations necessary to observe pairs. This broadening is due to interactions with nonnearest neighbor guests and with other impurities. 5 We do not believe that "curve fitting" would have helped significantly our measurements of splittings, as we do not feel that we have a clear-cut theoretical understanding of the nature of the line shapes. The frequency of the interchange equivalent pair absorptions

TABLE II. Results of mixed crystal absorption.

\begin{tabular}{|c|c|c|c|c|}
\hline$\tilde{p}\left(\mathrm{~cm}^{-1}\right)$ & Conc, dep. ${ }^{2}$ & $\Delta \bar{\nu}$ & Polarization & Assignment \\
\hline \multicolumn{5}{|c|}{$1-\mathrm{DC}_{10} \mathrm{H}_{7}$ in $\mathrm{C}_{t 0} \mathrm{D}_{8}$} \\
\hline $31568 \pm 2$ & nonlinear & $18 \pm 2$ & $\| b$ & $1 / 2(a+b)$ pair \\
\hline $31558.0 \pm 0.5$ & linear & $8.0: 0.5$ & $\| b>1 b$ & $2-\mathrm{D}^{1 / 3} \mathrm{CC}_{9} \mathrm{H}_{3}$ \\
\hline $31556.3 \div 0.5$ & linear & $6.3: 0.5$ & $\| b>1 b$ & $2-\mathrm{DC}_{10} \mathrm{H}_{3}$ \\
\hline $31552,2 \div 0.5$ & linear & $2.2 \pm 0.5$ & $\| b>L b$ & $1-\mathrm{D}^{13} \mathrm{CC}_{9} \mathrm{H}_{7}$ \\
\hline $31550.0 \pm 0.5$ & linear & 0 & $\| b>\perp b$ & $1-\mathrm{DC}_{10} \mathrm{H}_{7}$ \\
\hline $31547.8 \pm 0.5$ & nonlinear & $-2.2 \pm 0.5$ & $A b>\perp b$ & pairb \\
\hline $31545.9 \pm 0.5$ & nonlinear & $-4.1 \pm 0.5$ & $\| b>\perp b$ & pair ${ }^{b}$ \\
\hline $31543.4 \pm 0.5$ & linear & $-6.6 \pm 0.5$ & $\| b>\perp b$ & ${ }^{13} \mathrm{CC}_{9} \mathrm{H}_{8}$ \\
\hline $31541.7 \pm 0.5$ & linear & $-8.3 \pm 0.5$ & $\| b>1 b$ & $\mathrm{C}_{10} \mathrm{H}_{8}$ \\
\hline $31535.9 \pm 0.5$ & nonlinear & $-14.1 \pm 0.5$ & $\perp b$ & $1 / 2(a+b)$ pair \\
\hline \multicolumn{5}{|c|}{$2-\mathrm{DC}_{10} \mathrm{H}_{7}$ in $\mathrm{C}_{10} \mathrm{D}_{8}$} \\
\hline $31572 \pm 1$ & nonlinear & $16 \pm 2$ & $\| b$ & $1 / 2(a+b)$ pair \\
\hline $31557.1 \pm 0.5$ & linear & $1.4 \pm 0.5$ & $\| b>1 b$ & $2-\mathrm{D}^{13} \mathrm{CC}_{9} \mathrm{H}_{3}$ \\
\hline $31555.7 \pm 0.5$ & linear & 0 & $\| b>\perp b$ & $2-\mathrm{DC}_{10} \mathrm{H}_{2}$ \\
\hline $31552.8 \pm 0.5$ & nonlinear & $-2.9 \pm 0.5$ & $\| b>1 b$ & pair ${ }^{6}$ \\
\hline $31550.7 \pm 0.5$ & nonlinear & $-5.0 \pm 0.5$ & $\| b>\perp b$ & pair ${ }^{b}$ \\
\hline $31549.1 \pm 0.5$ & linear & $-6.1 \pm 0.5$ & $\| b>\perp b$ & $1-\mathrm{DC}_{10} \mathrm{H}_{7}$ \\
\hline $31541.5 \pm 0.5$ & nonlinear & -1411 & $\| b \sim \perp b$ & $\begin{array}{l}\mathrm{C}_{10} \mathrm{H}_{8} \text { and } \\
1 / 2(a+b) \text { pair }\end{array}$ \\
\hline \multicolumn{5}{|c|}{$1,4-\mathrm{D}_{2} \mathrm{C}_{10} \mathrm{H}_{7}$ in $\mathrm{C}_{10} \mathrm{D}_{8}$} \\
\hline $31574 \pm 2$ & nonlinear & $17 \pm 2$ & $\| b$ & $1 / 2(a+b)$ pair \\
\hline $31561.3 \pm 0.5$ & nonlinear & $3.8 \pm 0.5$ & $\| b>1 b$ & pais ${ }^{b}$ \\
\hline $31559.1 \pm 0.5$ & linear & $1.6 \pm 0.5$ & $a>\perp b$ & $\mathrm{I}, 4-\mathrm{D}_{2}{ }^{13} \mathrm{CC}_{9} \mathrm{H}_{6}$ \\
\hline $31557.5 \pm 0.5$ & linear & 0 & $\| b>1 b$ & $1,4-D_{2} C_{10} H_{6}$ \\
\hline $31554.1 \pm 0.5$ & nonlinear & $-3.4 \pm 0.5$ & $\| b>1 b$ & pair $x^{b}$ \\
\hline $31552.5 \pm 0.5$ & nonlinear & $-5.4 \pm 0.5$ & $\| b>\perp b$ & pair ${ }^{b}$ \\
\hline $31549.5 \pm 0.5$ & linear & $-8.0 \pm 0.5$ & $\| b>\perp b$ & $1-\mathrm{DC}_{10} \mathrm{H}_{7}$ \\
\hline $31544.1=0.5$ & nonlinear & $-13.4 \pm 0.5$ & $\perp b$ & $1 / 2(a+b)$ pair \\
\hline $31541.0 \pm 0.5$ & linear & $-14.5 \pm 0.5$ & $\| b>\perp b$ & $\mathrm{C}_{10} \mathrm{H}_{8}$ \\
\hline
\end{tabular}

a Concentration dependence of absorption intensity relative to the monomer.

b' Pair" without designation indicates that the transition is due to a translationally equivalent pair. 

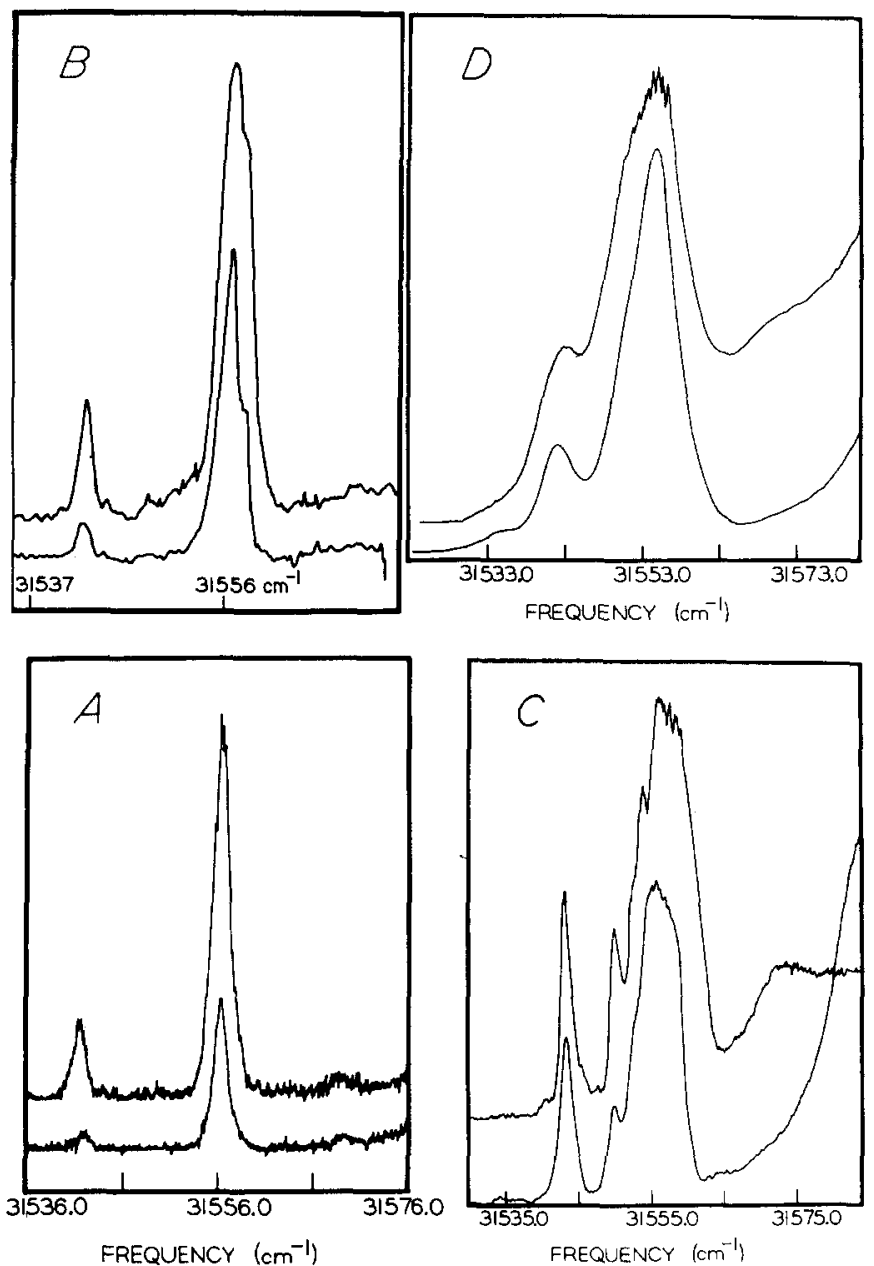

FIG. 2, 2-DC ${ }_{10} \mathrm{H}_{7}$ in $\mathrm{C}_{10} \mathrm{D}_{8}$ absorption spectra. In each figure the $\| \mathrm{b}$ polarized spectrum is above the $\perp \mathrm{b}$. Each spectrum was recorded photoelectrically with a resolution of $0.7 \mathrm{~cm}^{-1}$. The concentration of $\beta \mathrm{D}_{1}$ is $0.0092 \%, 0.14 \%, 0.91 \%$, and $4.9 \%$ mole in $a-d$, respectively.

should be better determined. However, in the b polarized transition the width of the peak makes calibration here relatively uncertain as well. The refreshing exception is the $\perp b$ interchange line which is sharp and well separated from the monomer line. However, it is also complicated by its frequent habit of lying close to another guest in the $\alpha \mathrm{D}_{2}$ and $\beta \mathrm{D}_{1}$ spectra.

\section{PARAMETER TESTING}

The principal aim of this investigation was to test and refine the three sets of interaction parameters determined by Hong and Kopelman ${ }^{5}$ from Hanson's ${ }^{3}$ data, and possibly remove assignment ambiguities. However, when the theoretical splittings were calculated from a program written by Hong, it became clear that the predicted difference in dimer splittings among the various sets, as the trap depth was decreased, was much smaller than the confidence limits on our calibration. These predicted splittings are shown in Table III under the headings "Set 1 " etc. The pair to which each splitting corresponds is indicated to the right of the calculated splitting. These calculations clarify why some of the dimer transitions went unobserved. In the three cases we investigated, an expected transition at about $-8 \mathrm{~cm}^{-1}$ from the monomer was unobserved. Even though this transition is well separated from the monomer, it would be very close to the impurity located at about $-8 \mathrm{~cm}^{-1}$ in each of the three systems (Table II). The transition expected at about $3-4 \mathrm{~cm}^{-1}$ above the monomer falls very close to the ${ }^{13} \mathrm{C}$ substituted monomer, making its observation more difficult (Table II). As can be seen by comparing the values of the predicted splittings (Table III), the largest change (as a function of the set of parameters) is expected for the $b$ polarized interchange pair. However, as pointed out above, the absorption peak corresponding to this transition is of a broad nature and hence there is a large uncertainty in its position. All translational pair variations are on the order of $0.5 \mathrm{~cm}^{-1}$ or less, which is equal to or less than the uncertainty in the calibration of the corresponding transitions. The only possibly useful results are those for the $\perp \mathbf{b}$ interchange pair results of $\alpha \mathrm{D}_{1}$ and $\alpha \mathrm{D}_{2}$. These seem to indicate that sets 2 and 3 are better. However, Hanson's pair data ${ }^{3}$ for $\mathrm{H}_{8}$ seems to point ${ }^{5}$ to set 1 as the preferred set; therefore even this criterion seems ambiguous. We doubt that
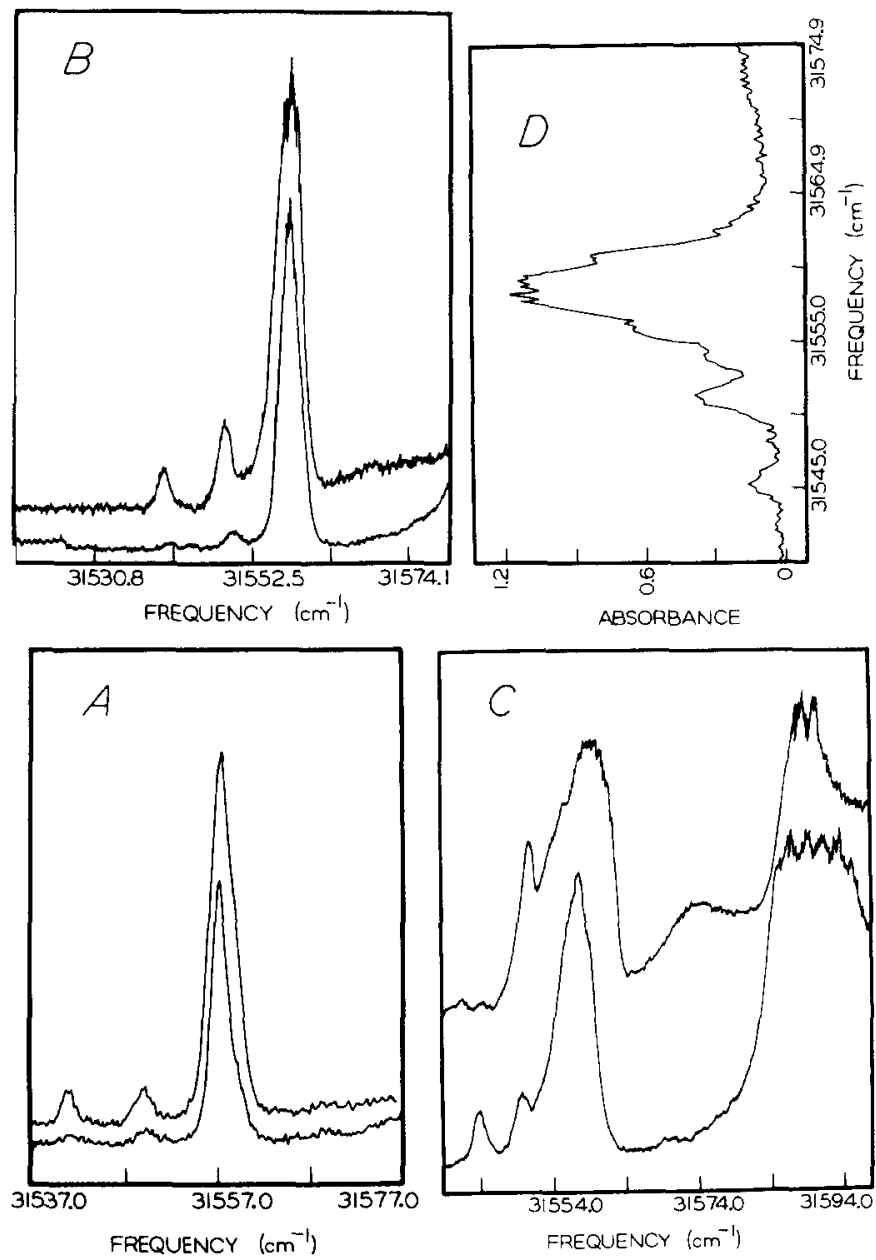

FIG. 3. Absorption of $1,4-\mathrm{D}_{2} \mathrm{C}_{10} \mathrm{H}_{6}$ in $\mathrm{C}_{10} \mathrm{D}_{8}$. Parts $a-c$ were recorded photoelectrically with a resolution of $0.7 \mathrm{~cm}^{-1}$. Part $d$ was recorded in third order with a resolution of about $0.4 \mathrm{~cm}^{-1}$. The first three are polarized with the $\| \mathrm{b}$ polarization above the $\perp \mathrm{b}$. The concentration of $\alpha \mathrm{D}_{2}$ in $a$ is $0.07 \%$ mole; in $b, 0.22 \%$ mole; and in $c$ and $d, 1.0 \%$ mole. 
TABLE III. Comparison of observed and predicted resonance pair splittings. ${ }^{2}$

\begin{tabular}{|c|c|c|c|c|}
\hline & Expt. & Set 1 & Set 2 & Set 3 \\
\hline $\mathrm{H}_{8}{ }^{\mathrm{c}}$ & $\begin{array}{r}3.7 \\
-3.3 \\
-5.1 \\
-7.9 \\
-15.3\end{array}$ & $\begin{array}{l}21.71 / 2(a+b)^{b} \\
4.0 c \\
-3.5 a+c \\
-5.0 a \\
-8.0 b \\
-15.01 / 2(a+b)\end{array}$ & $\begin{array}{c}20.01 / 2(a+b) \\
4.0 a+c \\
-3.2 b \\
-5.2 c \\
-7.5 a \\
-14.71 / 2(a+b)\end{array}$ & $\begin{array}{l}19.01 / 2(a+b) \\
4.1 a+c \\
-3.4 b \\
-4.8 a \\
-7.9 c \\
-14.21 / 2(a+b)\end{array}$ \\
\hline$\alpha \mathrm{D}_{1}$ & $\begin{array}{l}18 . \pm 2 \\
\cdots \\
-2.2 \pm 0.5 \\
-4.1 \pm 0.5 \\
\ldots . \\
-14.1 \pm 0.5\end{array}$ & $\begin{array}{l}21.51 / 2(a+b) \\
3.8 c \\
-3.7 a+c \\
-5.2 a \\
-7.8 b \\
-14.51 / 2(a+b)\end{array}$ & $\begin{array}{l}20.51 / 2(a+b) \\
3.7 a+c \\
-3.6 b \\
-5.1 c \\
-7.5 a \\
-14.51 / 2(a+b)\end{array}$ & $\begin{array}{l}19.31 / 2(a+b) \\
3.6 a+c \\
-3.3 b \\
-4.8 a \\
-7.8 c \\
-13.71 / 2(a+b)\end{array}$ \\
\hline$\beta \mathrm{D}_{1}$ & $\begin{array}{l}16 . \pm 2 \\
\cdots \\
-2.9 \pm 0.5 \\
-5.0 \pm 0.5 \\
\cdots \\
-14 . \pm 1\end{array}$ & $\begin{aligned} 21.51 / 2(a+b) \\
3.5 c \\
-3.7 a+c \\
-5.5 a \\
-7.7 b \\
-14.01 / 2(a+b)\end{aligned}$ & $\begin{array}{l}20.61 / 2(a+b) \\
3.5 a+c \\
-3.7 b \\
-5.0 c \\
-7.5 a \\
-13.81 / 2(a+b)\end{array}$ & $\begin{array}{l}19.41 / 2(a+b) \\
3.5 a+c \\
-3.2 b \\
-4.8 a \\
-7.5 c \\
-13.51 / 2(a+b)\end{array}$ \\
\hline$\alpha D_{2}$ & $\begin{aligned} & 17 . \pm 2 \\
& 3.8 \pm 0.5 \\
&-3.5 \pm 0.5 \\
&-5.4 \pm 0.5 \\
& \cdots \\
&-13.4 \\
&\end{aligned}$ & $\begin{aligned} 21.51 / 2(a+b) \\
3.5 c \\
-3.7 a+c \\
-5.5 a \\
-7.6 b \\
-14.01 / 2(a+b)\end{aligned}$ & $\begin{aligned} 20.61 / 2(a+b) \\
3.5 a+c \\
-3.7 b \\
-5.0 c \\
-7.5 a \\
-13.71 / 2(a+b)\end{aligned}$ & $\begin{array}{l}19.51 / 2(a+b) \\
3.5 a+c \\
-3.2 b \\
-4.6 a \\
-7.5 c \\
-13.01 / 2(a+b)\end{array}$ \\
\hline
\end{tabular}

${ }^{2}$ Expressed in $\mathrm{cm}^{-1}$.

${ }^{\mathrm{b}}$ These refer to the orientation of the second molecule in the dimer relative to the first.

'Hanson (Ref. 3).

this situation can be improved by further investigation of dimer splittings even at higher resolution because of the small magnitude of predicted changes and the difficulties with interference from other guests and bandwidth problems (as pointed out above).

A second important point is that the variation in pair splitting predicted by each set of parameters with varying trap depth is very small for all of the pair transitions except for the $\perp \mathrm{b}$ interchange pair transition. However, this transition seems definitely to show the general trend calculated from the superexchange theory. (Of course, the absolute change here again is quite small.) We thus conclude that our results do substantiate the theory provided by Hong and Kopelman. ${ }^{5}$ It is unfortunate, however, that the experiments are not very useful at this point in allowing one to determine the best of the three sets of interaction energies. It may be possible to carry out this determination by investigation of interchange equivalent resonance "trimers." A transition probably due to this state has been reported by Mauser, Port, and Wolf ${ }^{19}$ and by Hong and Robinson ${ }^{12}$ in fluorescence spectra of $H_{8}$ in $D_{8}$ mixed crystals. This transition is located at about $22 \mathrm{~cm}^{-1}$ below the monomer transition. Unfortunately the work done so far has produced rather complex spectra due to the high concentrations used. However, it should be possible to observe these states at lower concentrations in reasonably thick crystals. The advantages of observing these transitions are (1) that the energy is in a range that should have little interference from other isotopic impurities (2) that the splitting should take on the characteristics of the trap depth dependence of the $\perp \mathbf{b}$ interchange pair (which is the greatest variation as mentioned above), and ( 3 ) that a greater interaction with the host might be expected since the species making up the state is $50 \%$ larger than is a pair state. The main difficulty will be that there are different configurations of guest trimers and this might complicate the situation. A theoretical superexchange calculation for trimers and tetramers is now feasible, following work on benzene. ${ }^{20}$

\section{DISCUSSION AND CONCLUSIONS}

Combining the total data on the naphthalene first singlet, i.e., both the monomer ${ }^{1}$ and resonance pair (above and mixed dimers ${ }^{6}$ ), one is tempted to argue that "set 2" gives the overall best fit. Conservatively speaking though, it may be that none of the sets adequately accounts for all the experimental splittings. However, the more significantly outstanding fact is the fairly close agreement of all three sets with a large amount of experimental data. If we consider the experimental exciton density of states ${ }^{16}$ to represent about six data points (including Davydov components), the monomer energies ${ }^{1}$ seven, the Rashba effect ${ }^{1}$ six, the monomer localization data ${ }^{1}$ five, the resonance pairs 18 energies (Tables II, III), and about 10 (Table II) polarizations, in addition to about 10 trimer data, 6,21 we have a total of about 60 data bits fitted by six pairwise interaction parameters.

The fact that two or even three sets can fit all the above data may be due to some lack of precision remaining in the experimental data. However, we may also be approaching a theoretical limitation owing to factors excluded from the present theory, like isotope effects on site shifts and orientation effects, ${ }^{1}$ which may be related to differences in the "phonon dressing" of the excitations (mass effect on exciton phonon coupling), as well as a lack in exact interchange symmetry of the resonance pairs. ${ }^{6}$ In addition, there is the obvious problem of neglecting longer range interactions. One can add to this list vibronic renormalization (which is isotope dependent) and its effects on configuration interaction.

In view of the above list of potential "complications" one should rather be content with the success of our simple minded theory. We have put our basic concepts, ${ }^{7}$ our exciton density-of-states, ${ }^{3-5}$ and our oversimplified exciton dispersion relations ${ }^{4,5}$ for naphthalene $\left({ }^{1} B_{2 u}\right)$ to a stringent test and we have met it within essentially $1 \mathrm{~cm}^{-1}$.

\footnotetext{
*Supported by NIH Grant NS08116.

tPresent address: 2849 Esmond Avenue, Richmond, CA 94804. ${ }^{1} \mathrm{~F}$. W. Ochs, P. N. Prasad, and R. Kopelman, Chem. Phys. 6, 253 (1974).

${ }^{2}$ R. Kopelman, J. Chem. Phys. 47, 3227 (1967).

${ }^{3}$ D. M. Hanson, J. Chem. Phys. 52, 3409 (1970).

${ }^{4} \mathrm{H}-\mathrm{K}$. Hong and R. Kopelman, Phys. Rev. Lett. 25, 1030 (1970).

${ }^{5}$ H-K. Hong and R. Kopelman, J. Chem. Phys. 55, 724 (1971).

${ }^{6} \mathrm{H}-\mathrm{K}$. Hong and R. Kopelman, J. Chem. Phys. 57, 3888 (1972).

${ }^{7}$ R. Kopelman, in Excited States, edited by E. C. Lim, (Aca-
} 
demic, New York, 1975), Vol. II.

${ }^{8}$ S. D. Colson, R. Kopelman, and G. W. Robinson, J. Chem. Phys. 47, 27, 5462 (1967).

${ }^{9}$ R. Kopelman and J. C. Laufer, in Electronic Density of State's, edited by L. H. Bennett, Nati. Bur. Stands.

(U.S.G. P. O., Washington, D. C., 1971).

${ }^{1}$ 'J. Hoshen, R. Kopelman, and J. Jortner, Chem. Phys. 10, 185 (1975).

${ }^{11}$ V. L. Broude and A. V. Leiderman, JETP Lett, 13, 302 (1971).

${ }^{12} \mathrm{H}-\mathrm{K}$. Hong and G. W. Robinson, J. Chem. Phys. 54, 1369 (1971).

${ }^{12}$ C. L. Braun and H. C. Wolf, Chem. Phys. Lett. 9, 260 (1971).
${ }^{14}$ E. R. Bernstein, S. D. Colson, R. Kopelman, and G. W. Robinson, J. Chem. Phys. 48, 5596 (1968).

${ }^{15}$ D. M. Hanson, R. Kopelman, and G. W. Robinson, J. Chem. Phys. 51, 212 (1969).

${ }^{16} \mathrm{~S}$. D. Colson, D. M. Hanson, R. Kopelman, and G.W. Robinson, J. Chem. Phys, 48, 2215 (1968).

${ }^{17} \mathrm{~F}$. Yonezawa and T. Matsubara, Prog. Theor. Phys, (Kyoto) 35, 357, 759 (1966).

${ }^{18}$ R. Kopelman, J. Chem. Phys. 47, 2631 (1967)。

${ }^{19}$ K. E. Mauser, H. Port, and H. C. Wolf, Chem. Phys, 1, 74 (1973).

${ }^{20} \mathrm{~J}$. Hoshen and R. Kopelman (unpublished).

${ }^{21} \mathrm{H}-\mathrm{K}$. Hong and R. Kopelman, J. Chem. Phys. 55, 5380 (1971). 